\title{
PRINCIPAIS ALTERAÇÕES DO HEMOGRAMA POTENCIALMENTE ASSOCIADAS AO COVID-19
}

\section{Brenda Zimmer Rohde ${ }^{1}$; Giane Engel Montagner ${ }^{2}$; Anna Karolline Rubim Rodrigues Oviedo ${ }^{3}$; Alencar Kolinski Machado4; Solange Binotto Fagann; Patrícia Gomes 6}

\section{RESUMO}

O presente trabalho teve como objetivo realizar uma revisão de literatura de artigos de pesquisa relacionados à parâmetros presentes no hemograma completo em pacientes com a doença COVID-19. A estratégia de busca usou strings de busca construídas e adaptadas para três bancos de dados eletrônicos: Web of Science, Scopus e PubMed, sendo elas "COVID-19 AND blood-count AND cohort". Segundo os resultados de eritrócitos, hemoglobina, hematócritos, HCM, VCM, RDW, leucócitos, neutrófilos, eosinófilos, monócitos, linfócitos e plaquetas consegue-se ter parâmetros para avaliar pacientes com COVID-19 e ter um prognóstico de como a doença irá progredir no paciente. O presente trabalho conclui que os parâmetros: hemoglobina, VCM, RDW, leucócitos, neutrófilos, monócitos, linfócitos e plaquetas apresentaram resultados importantes para avaliar e realizar prognóstico em pacientes com COVID19.

Palavras-chave: Eritrograma; Leucograma; Plaquetograma; SARS-CoV-2.

Eixo Temático: Atenção Integral e Promoção à Saúde

\footnotetext{
1 Brenda Zimmer Rohde - Universidade Franciscana. brenda.rohde@ufn.edu.br

${ }^{2}$ Giane Engel Montagner - Universidade Franciscana. giane.engel@ufn.edu.br

${ }^{3}$ Anna Karolline Oviedo - Universidade Franciscana. annakarollineoviedo@gmail.com

${ }^{4}$ Alencar Kolinski Machado - Universidade Franciscana. alencar.machado@ufn.edu.br

${ }^{5}$ Solange Binotto Fagan - Universidade Franciscana. sfagan@ufn.edu.br

${ }^{6}$ Patrícia Gomes - Universidade Franciscana. patriciagomes@ufn.edu.br
} 


\section{INTRODUÇÃO}

A propagação global da síndrome respiratória aguda grave coronavírus 2 (SARS-CoV-2) gerou um surto de pandemia no final de 2019 - início de 2020, que teve um impacto severo na prestação de cuidados em todo o mundo. O quadro clínico causado pela infecção viral acima mencionada foi chamada de Coronavírus doença 2019 (COVID-19): suas manifestações clínicas abrangem uma ampla gama de entidades, desde uma doença semelhante à gripe leve até formas com risco de vida (BELLAN et al., 2021). Vários estudos observacionais têm mostrado que resposta imune-inflamatória e tempestade de citocinas podem ser responsáveis pela falência de múltiplos órgãos e progressão fatal de COVID-19 (SAYAH et al., 2021). O presente trabalho teve como objetivo realizar uma revisão de literatura de artigos de pesquisa relacionados à parâmetros presentes no hemograma completo em pacientes com a doença COVID-19.

\section{METODOLOGIA}

Este estudo caracteriza-se como exploratório, do tipo revisão integrativa da literatura. Essa revisão recuperou e avaliou os dados disponíveis em bancos de dados sobre a relação do hemograma em pacientes com COVID-19. A estratégia de busca usou strings de busca construídas e adaptadas para os bancos de dados: Web of Science, Scopus e PubMed, sendo elas "COVID-19 AND blood-count AND cohort".

A busca avançada nas bases de dados foi realizada por pares, considerando artigos de pesquisa publicados até 14 de setembro de 2021, onde os autores conduziram a seleção preliminar e extração de dados de forma independente, na etapa de identificação e após prosseguiram para as etapas de screening e inclusão, levando em consideração os critérios de inclusão e exclusão. Foram incluídos estudos nas línguas inglesa e espanhola que realizaram estudos de coorte que verificaram a correlação de parâmetros do hemograma com a COVID-19, em adultos. Foram excluídos estudos de revisão, cartas ao editor, notas, artigos duplicados, estudos com menores de 18 anos e que não correspondem aos objetivos do estudo. 
Para a extração de dados dos artigos incluídos neste estudo, os autores extraíram e resumiram independentemente as seguintes informações: parâmetros do hemograma analisados, número de pacientes, parâmetros não alterados, parâmetros alterados e referência.

\section{RESULTADOS E DISCUSSÕES}

A busca nas bases de dados resultou na coleta de 115 artigos, sendo 26 na Web of Science, 36 na Scopus e 53 na PubMed. Destes, 22 atenderam adequadamente aos critérios de inclusão e foram selecionados para esta revisão. $A$ Figura 1 mostra o fluxograma com a estratégia de seleção dos artigos incluídos.

Figura 1 - Fluxograma com a estratégia de seleção de artigos.

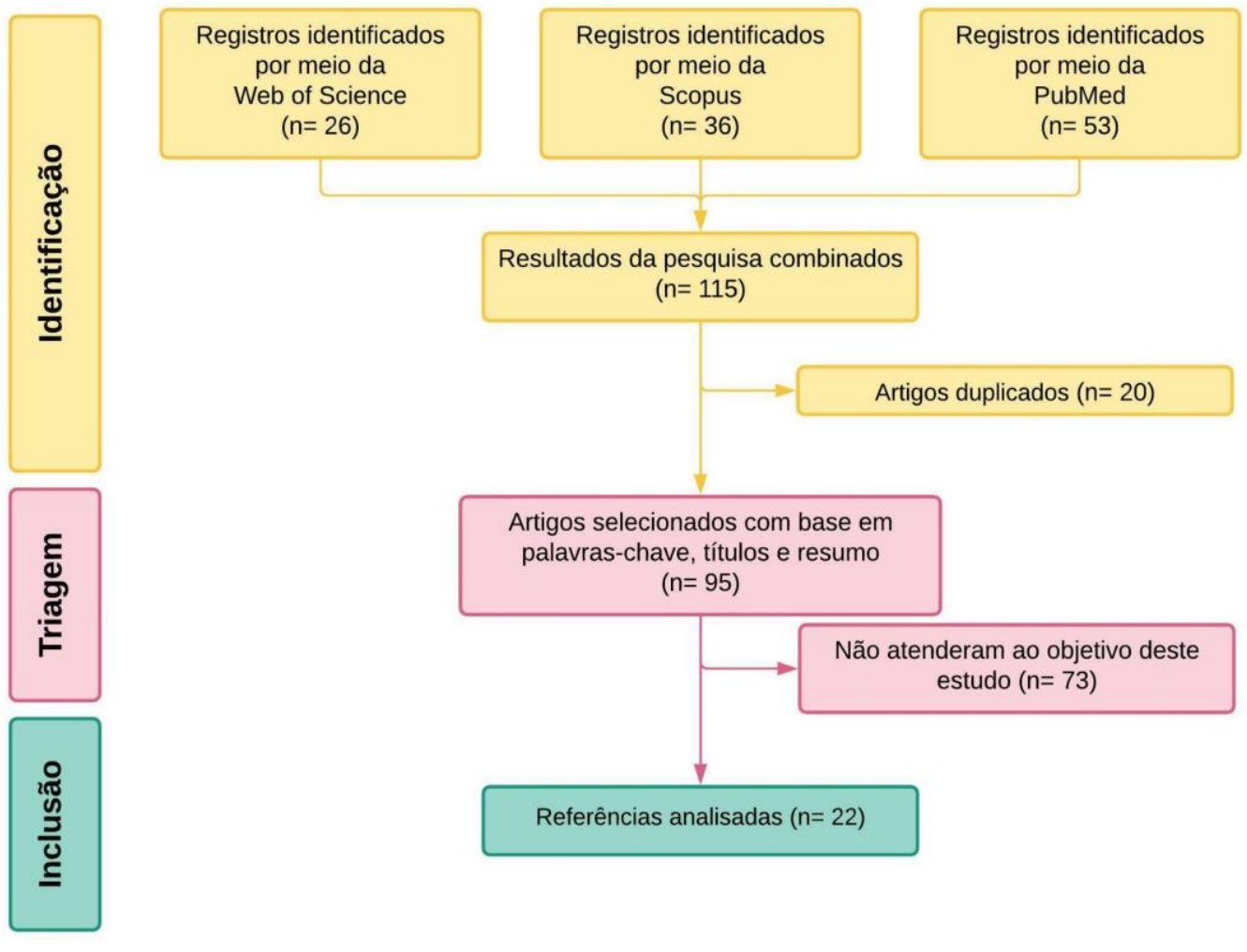

No Quadro 1 se encontram os parâmetros dos hemogramas analisados, seguido do número de pacientes avaliados no estudo e suas possíveis alterações em pacientes positivos e negativos para COVID-19. 
Quadro 1 - Estudos que comparativos de pacientes positivos e negativos para COVID-19.

\begin{tabular}{|c|c|c|c|c|}
\hline $\begin{array}{l}\text { Parâmetros do } \\
\text { hemograma } \\
\text { analisados }\end{array}$ & $\begin{array}{c}\text { № de } \\
\text { pacientes }\end{array}$ & $\begin{array}{l}\text { Parâmetros não } \\
\text { alterados }\end{array}$ & $\begin{array}{l}\text { Parâmetros } \\
\text { alterados }\end{array}$ & Referência \\
\hline $\begin{array}{c}\text { Hemoglobina, } \\
\text { Leucócitos, VCM, } \\
\text { RNL, RPL, RLM, RME, } \\
\text { RNM, RDW, Plaquetas }\end{array}$ & 301 & $\begin{array}{l}\text { Leucócitos, RNL, } \\
\text { RPL, RLM, RNM, } \\
\text { RDW, Plaquetas }\end{array}$ & $\begin{array}{c}\text { Hemoglobina, VCM, } \\
\text { RME }\end{array}$ & $\begin{array}{l}\text { Akman e } \\
\text { Bakirdogen } \\
(2021)\end{array}$ \\
\hline $\begin{array}{l}\text { Hemoglobina, } \\
\text { Leucócitos, } \\
\text { Neutrófilos, Linfócitos, } \\
\text { Plaquetas, RNL, RPL }\end{array}$ & 1054 & - & $\begin{array}{l}\text { Hemoglobina, } \\
\text { Leucócitos, } \\
\text { Neutrófilos, } \\
\text { Linfócitos, } \\
\text { Plaquetas, RNL, } \\
\text { RPL }\end{array}$ & $\begin{array}{l}\text { Eslamijouybari } \\
\text { et al. }(2020)\end{array}$ \\
\hline $\begin{array}{l}\text { Linfócitos, RNL, } \\
\text { Plaquetas, } \\
\text { Hemoglobina }\end{array}$ & 80 & Plaquetas & Linfócitos, RNL & $\begin{array}{l}\text { Nalbant et al. } \\
\quad(2020)\end{array}$ \\
\hline $\begin{array}{c}\text { Eosinófilos, Linfócitos, } \\
\text { Hemoglobina, } \\
\text { Plaquetas }\end{array}$ & 121 & $\begin{array}{l}\text { Hemoglobina, } \\
\text { Plaquetas }\end{array}$ & $\begin{array}{l}\text { Eosinófilos, } \\
\text { Linfócitos }\end{array}$ & $\begin{array}{l}\text { Outh et al. } \\
\text { (2021) }\end{array}$ \\
\hline $\begin{array}{c}\text { Eritrócitos, } \\
\text { Hemoglobina, VCM, } \\
\text { HCM, CHCM, } \\
\text { Plaquetas, Leucócitos, } \\
\text { Neutrófilos, Linfócitos, } \\
\text { Monócitos, RNL }\end{array}$ & 951 & $\mathrm{CHCM}$ & $\begin{array}{l}\text { Eritrócitos, } \\
\text { Hemoglobina, VCM, } \\
\text { HCM, Plaquetas, } \\
\text { Leucócitos, } \\
\text { Neutrófilos, } \\
\text { Linfócitos, } \\
\text { Monócitos, RNL }\end{array}$ & $\begin{array}{l}\text { Sayed et al. } \\
\quad(2021)\end{array}$ \\
\hline $\begin{array}{c}\text { Hemoglobina, } \\
\text { Hematócrito, } \\
\text { Eosinófilo, Neutrófilo, } \\
\text { Linfócito, Plaqueta, } \\
\text { RDW, VCM, RPL, RNL }\end{array}$ & 233 & $\begin{array}{l}\text { Hemoglobina, } \\
\text { Hematócrito, } \\
\text { Neutrófilo, RDW, } \\
\text { VCM }\end{array}$ & $\begin{array}{l}\text { Eosinófilo, Linfócito, } \\
\text { Plaqueta, RPL, RNL }\end{array}$ & $\begin{array}{l}\text { Seyit et al. } \\
\quad(2021)\end{array}$ \\
\hline
\end{tabular}

VCM - Volume Corpuscular Médio; HCM - Hemoglobina Corpuscular Média; CHCM - Concentração Média de Hemoglobina Corpuscular; RDW - Red Cell Distribution Width; RNL - Relação Neutrófilo para Linfócito; RPL - Relação Plaqueta para linfócito; RLM - Relação Linfócito para Monócito; RME - Relação Monócito para Eosinófilo; RNM - Relação Neutrófilo para Monócito.

No Quadro 2 se encontram os parâmetros dos hemogramas analisados, seguido do número de pacientes avaliados no estudo e suas possíveis alterações em pacientes graves com não graves com COVID-19. 
Quadro 2 - Estudos que comparativos de pacientes graves e não graves com COVID-19.

\begin{tabular}{|c|c|c|c|c|}
\hline $\begin{array}{l}\text { Parâmetros do } \\
\text { hemograma } \\
\text { analisados }\end{array}$ & $\begin{array}{c}\text { № de } \\
\text { pacientes }\end{array}$ & $\begin{array}{l}\text { Parâmetros não } \\
\text { alterados }\end{array}$ & Parâmetros alterados & Referência \\
\hline $\begin{array}{l}\text { Leucócitos, Neutrófilos, } \\
\text { Linfócitos, Eosinófilos, } \\
\text { RNL, Plaquetas, } \\
\text { Hemoglobina, HCM, } \\
\text { VCM, RDW }\end{array}$ & 664 & Hemoglobina & $\begin{array}{l}\text { Leucócitos, Neutrófilos, } \\
\text { Linfócitos, Eosinófilos, } \\
\text { RNL, Plaquetas, HCM, } \\
\text { VCM, RDW }\end{array}$ & $\begin{array}{l}\text { Bellan et } \\
\text { al. }(2021)\end{array}$ \\
\hline Hemoglobina, RDW & 186 & - & Hemoglobina, RDW & $\begin{array}{c}\text { Bilgir et al. } \\
(2020)\end{array}$ \\
\hline $\begin{array}{c}\text { Eritrócitos, } \\
\text { Hematócritos, } \\
\text { Hemoglobina, VCM, } \\
\text { HCM, CHCM, RDW, } \\
\text { Leucócitos, Linfócitos, } \\
\text { Plaquetas } \\
\end{array}$ & 1641 & Leucócitos & $\begin{array}{l}\text { Eritrócitos, } \\
\text { Hematócritos, } \\
\text { Hemoglobina, VCM, } \\
\text { HCM, CHCM, RDW, } \\
\text { Linfócitos, Plaquetas }\end{array}$ & $\begin{array}{l}\text { Foy et al. } \\
\text { (2020) }\end{array}$ \\
\hline $\begin{array}{l}\text { Plaquetas, Leucócitos, } \\
\text { Linfócitos, Neutrófilos, } \\
\text { RNL }\end{array}$ & 88 & $\begin{array}{c}\text { Plaquetas, } \\
\text { Linfócitos, RNL }\end{array}$ & Leucócitos, Neutrófilos & $\begin{array}{l}\text { Galland et } \\
\text { al. (2021) }\end{array}$ \\
\hline $\begin{array}{c}\text { Eritrócitos, } \\
\text { Hemoglobina, } \\
\text { Hematócrito, Plaquetas, } \\
\text { Leucócitos, Neutrófilos, } \\
\text { Linfócitos, Monócitos, } \\
\text { Eosinófilos }\end{array}$ & 430 & Plaquetas & $\begin{array}{c}\text { Eritrócitos, } \\
\text { Hemoglobina, } \\
\text { Hematócrito, Leucócitos, } \\
\text { Neutrófilos, Linfócitos, } \\
\text { Monócitos, Eosinófilos, }\end{array}$ & $\begin{array}{l}\text { Gerotziafas } \\
\text { et al. } \\
(2020)\end{array}$ \\
\hline $\begin{array}{c}\text { Hemoglobina, } \\
\text { Leucócitos, Linfócitos }\end{array}$ & 66 & Hemoglobina & Leucócitos, Linfócitos & $\begin{array}{l}\text { Ghweil et } \\
\text { al. (2020) }\end{array}$ \\
\hline $\begin{array}{c}\text { Eritrócitos, } \\
\text { Hemoglobina, } \\
\text { Hematócrito, VCM, } \\
\text { HCM, CHCM, RDW, } \\
\text { Leucócitos, Neutrófilos, } \\
\text { Linfócitos, Monócitos, } \\
\text { Eosinófilos, Plaquetas, } \\
\text { RNL, RMN }\end{array}$ & 97 & $\mathrm{HCM}, \mathrm{CHCM}$ & $\begin{array}{c}\text { Eritrócitos, } \\
\text { Hemoglobina, } \\
\text { Hematócrito, VCM, } \\
\text { RDW, Leucócitos, } \\
\text { Neutrófilos, Linfócitos, } \\
\text { Monócitos, Eosinófilos, } \\
\text { Plaquetas, RNL, RMN }\end{array}$ & $\begin{array}{l}\text { Kilercik et } \\
\text { al. (2021) }\end{array}$ \\
\hline $\begin{array}{c}\text { Neutrófilos, Linfócitos, } \\
\text { Monócitos, Eritrócitos, } \\
\text { Hemoglobina, VCM, } \\
\text { RDW, Plaquetas }\end{array}$ & 379 & Monócitos, & $\begin{array}{c}\text { Neutrófilos, Linfócitos, } \\
\text { Eritrócitos, } \\
\text { Hemoglobina, VCM, } \\
\text { RDW, Plaquetas }\end{array}$ & $\begin{array}{l}\text { Lanini et al. } \\
\quad(2020)\end{array}$ \\
\hline $\begin{array}{l}\text { Leucócitos, Neutrófilos, } \\
\text { Linfócitos, RNL, } \\
\text { Monócitos, Eosinófilos, } \\
\text { Eritrócitos, } \\
\text { Hemoglobina, VCM, } \\
\text { Plaquetas }\end{array}$ & 923 & $\begin{array}{l}\text { Eosinófilos, VCM, } \\
\text { Plaquetas }\end{array}$ & $\begin{array}{l}\text { Leucócitos, Neutrófilos, } \\
\text { Linfócitos, RNL, } \\
\text { Monócitos, Eritrócitos, } \\
\text { Hemoglobina }\end{array}$ & $\begin{array}{l}\text { Linssen et } \\
\text { al. (2020) }\end{array}$ \\
\hline $\begin{array}{l}\text { Plaquetas, Leucócitos, } \\
\text { Neutrófilos, RNL, } \\
\text { Linfócitos, Hemoglobina }\end{array}$ & 12.759 & $\begin{array}{l}\text { Linfócitos, } \\
\text { Hemoglobina }\end{array}$ & $\begin{array}{l}\text { Plaquetas, Leucócitos, } \\
\text { Neutrófilos, RNL }\end{array}$ & $\begin{array}{l}\text { Liu et al. } \\
\text { (2021) }\end{array}$ \\
\hline
\end{tabular}




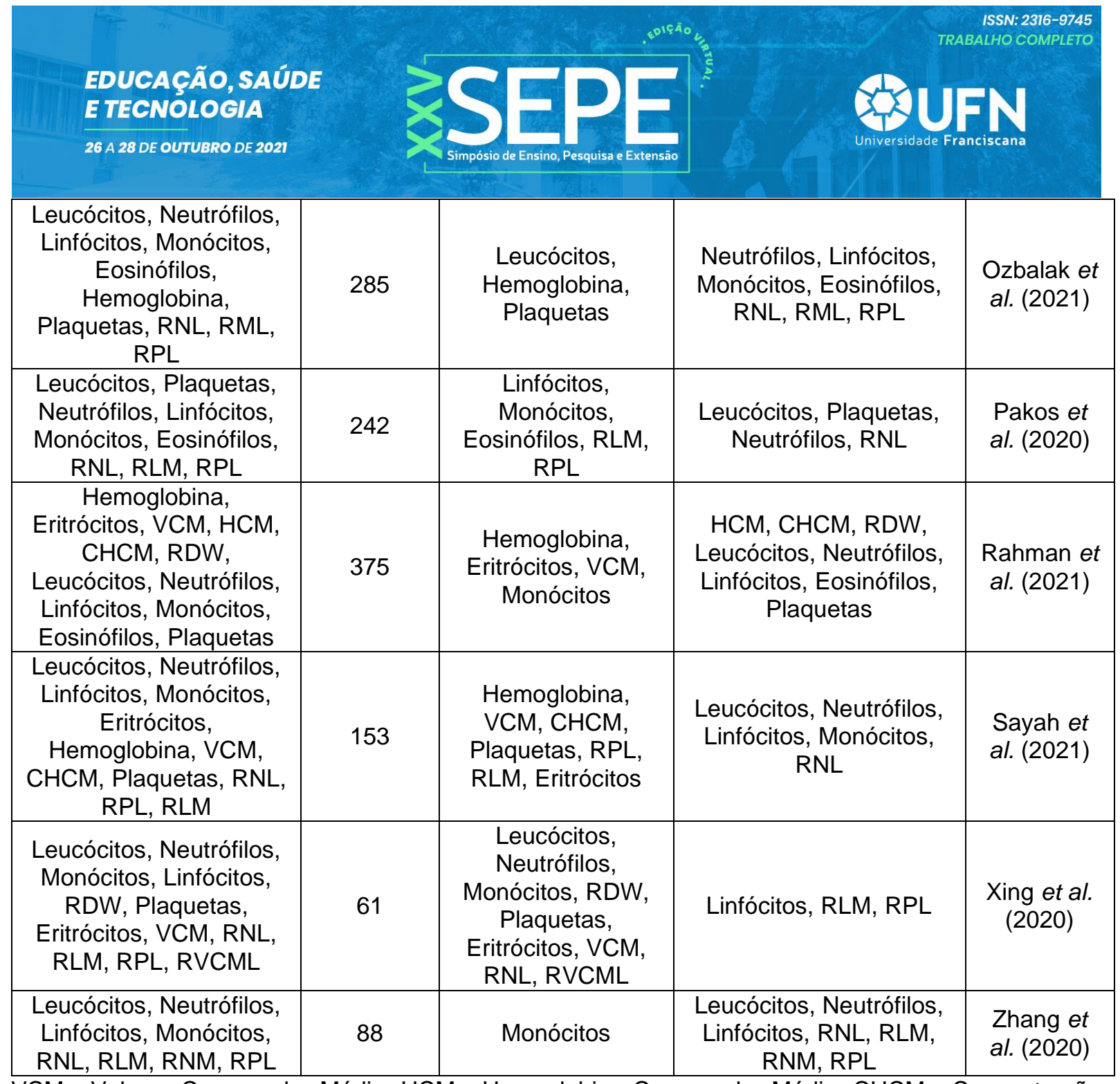

VCM - Volume Corpuscular Médio; HCM - Hemoglobina Corpuscular Média; CHCM - Concentração Média de Hemoglobina Corpuscular; RDW - Red Cell Distribution Width; RNL - Relação Neutrófilo para Linfócito; RPL - Relação Plaqueta para linfócito; RLM - Relação Linfócito para Monócito; RNM - Relação Neutrófilo para Monócito; RML - Relação Monócito para Linfócito; RVCML - Relação VCM para Linfócito.

\subsection{ERITROGRAMA}

O valor de eritrócitos foi encontrado reduzido em pacientes com COVID-19 em relação à pacientes sem a doença (SAYED et al., 2021) e também em 5 estudos de 8 que compararam pacientes graves com casos leves, onde indivíduos com casos graves de COVID-19 tiveram uma redução no número de glóbulos vermelhos em relação a casos leves. (FOY et al., 2020; GEROTZIAFAS et al., 2020; KILERCIK et al., 2021; LANINI et al., 2020; LINSSEN et al., 2020).

Houve uma diferença significativa entre os grupos com COVID-19 e sem a doença, em termos de hemoglobina, sendo o nível de hemoglobina menor em pacientes com COVID-19 (AKMAN e BAKIRDOGEN, 2021; ESLAMIJOUYBARI et al. 
2020; SAYED et al., 2021). Todos os atigos analisados $(n=6)$ que compararam pacientes graves com casos leves, onde indivíduos com casos graves de COVID-19 tiveram uma redução no número de hemoglobina em relação a casos leves. (BILGIR et al., 2021; GEROTZIAFAS et al., 2020; KILERCIK et al., 2021; LANINI et al., 2020; LINSSEN et al., 2020; FOY et al., 2020), bem como redução no número de hematócritos em relação a casos leves (KILERCIK et al., 2021; FOY et al., 2020; GEROTZIAFAS et al., 2020).

O HCM apresentou-se reduzido de pacientes com COVID-19 em relação aos pacientes sem a doença (SAYED et al., 2021). Em todos os estudos analisados ( $\mathrm{n}=$ 3) que compararam pacientes graves com casos leves, observou que indivíduos com casos graves de COVID-19 tiveram um aumento no HCM em relação a casos leves. (BELLAN et al., 2021; FOY et al., 2020; RAHMAN et al. 2021), bem como tiveram um aumento no CHCM em relação a casos leves (FOY et al., 2020; RAHMAN et al. 2021).

O VCM apresentou resultados significativos com níveis mais baixos de pacientes com COVID-19 em relação aos pacientes sem a doença (AKMAN E BAKIRDOGEN, 2021; SAYED et al., 2021). Em todos os estudos análisados $(n=2)$ que compararam pacientes graves com casos leves, observou que indivíduos com casos graves de COVID-19 tiveram um aumento no VCM em relação a casos leves (BELLAN et al., 2021; FOY et al., 2020; KILERCIK et al., 2021; LANINI et al., 2020).

Em todos os estudos analisados $(n=6)$ que compararam pacientes graves com casos leves, observou que indivíduos com casos graves de COVID-19 tiveram um aumento no RDW em relação a casos leves (BELLAN et al., 2021; BILGIR et al., 2021; FOY et al., 2020; KILERCIK et al., 2021; LANINI et al., 2020; RAHMAN et al., 2021). O significado preditivo de RDW no contexto de COVID-19 pode refletir o efeito sobre a eritropoiese de insuficiência respiratória e inflamação sistêmica (BELLAN et al., 2021). Bilgir e colaboradores (2020) sugerem um valor de corte de $15 \%$ e acima para RDW parece significativo em termos de mortalidade.

Segundo os resultados de eritrócitos, hemoglobina, hematócritos, HCM, VCM e RDW consegue-se ter parâmetros para avaliar e realizar prognóstico em pacientes com COVID-19.

\subsection{LEUCOGRAMA}


Os leucócitos apresentam resultados significativos com níveis mais elevados em pacientes com COVID-19 em relação aos pacientes sem a doença (ESLAMIJOUYBARI et al., 2020; SAYED et al., 2021). Em 7 estudos de 11 que compararam pacientes graves com casos leves, observou que indivíduos com casos graves de COVID-19 tiveram um aumento nos níveis de leucócitos em relação a casos leves (BELLAN et al., 2021; GALLAND et al., 2021; LIU et al., 2021; PAKOS et al., 2020; RAHMAN et al., 2021; SAYAH et al., 2021; ZHANG et al., 2020).

Os neutrófilos apresentam-se aumentados em pacientes com COVID-19 em relação aos pacientes sem a doença (ESLAMIJOUYBARI et al., 2020; SAYED et al., 2021). Em 11 estudos de 12 que compararam pacientes graves com casos leves, observou que indivíduos com casos graves de COVID-19 tiveram um aumento nos níveis de neutrófilos em relação a casos leves (BELLAN et al., 2021; GALLAND et al., 2021; GEROTZIAFAS et al., 2020; KILERCIK et al., 2021; LANINI et al., 2020; LINSSEN et al., 2020; ZHANG et al., 2020; LIU et al., 2021; OZBALAK et al., 2021; PAKOS et al., 2020; 2021; SAYAH et al., 2021).

Os neutrófilos desempenham seus papel protetor não só através da fagocitose, mas também explorando a formação de armadilha extracelular de neutrófilos (NET). Embora os NETs sejam benéficos na defesa contra patógenos, a formação sustentada de NET pode conduzir danos colaterais em diferentes condições humanas, incluindo infecções virais. Postula-se que, em COVID19, a produção inadequada de NET pode ser crucial no desenvolvimento da "tempestade de citocinas" responsável pela síndrome do desconforto respiratório (SDRA), inflamação grave síndrome de resposta (SIRS) e sepse (BELLAN et al., 2021).

Os eosinófilos apresentam resultados significativos com nível mais baixo em pacientes com COVID-19 em relação aos pacientes sem a doença (OUTH et al., 2021; SEYIT et al., 2021). Em 2 estudos de 3 que compararam pacientes graves com casos leves, observou que indivíduos com casos graves de COVID-19 tiveram uma diminuição nos níveis de eosinófilos em relação a casos leves (BELLAN et al., 2021; KILERCIK et al., 2021; RAHMAN et al., 2021). Os eosinófilos são úteis para distinguir pacientes de condições não infecciosas de condições infecciosas, especialmente infecções bacterianas (OUTH et al., 2021). 
Os monócitos apresentam resultados significativos com níveis mais baixos em pacientes com COVID-19 em relação aos pacientes sem a doença (SAYED et al., 2021). Em todos os estudos analisados $(n=5)$ que compararam pacientes graves com casos leves, observou que indivíduos com casos graves de COVID-19 tiveram uma diminuição nos níveis de monócitos em relação a casos leves (GEROTZIAFAS et al., 2020; KILERCIK et al., 2021; LINSSEN et al., 2020; OZBALAK et al., 2021; SAYAH et al., 2021). Macrófagos e monócitos são atores importantes na imunidade inata e adaptativa da resposta contra patógenos microbianos e virais por meio da produção de citocinas, atividade fagocítica e ativação de linfócitos. Muitos estudos concluíram que a pneumonia por COVID-19 está relacionada ao ataque de citocinas e à super ativação de monócitos e macrófagos (KILERCIK et al., 2021).

Os linfócitos apresentam resultados significativos com níveis menores em pacientes com COVID-19 em relação aos pacientes sem a doença (ESLAMIJOUYBARI et al., 2020; NALBANT et al., 2020; OUTH et al., 2021; SAYED et al., 2021; SEYIT et al., 2021). Em todos os estudos analisados ( $n=12)$ que compararam pacientes graves com casos leves, onde indivíduos com casos graves de COVID-19 tiveram uma diminuição nos níveis de linfócitos em relação a casos leves (BELLAN et al., 2021; FOY et al., 2020; GEROTZIAFAS et al., 2020; GHWEIl et al., 2020; KILERCIK et al., 2021; LANINI et al., 2020; LINSSEN et al., 2020; OZBALAK et al., 2021; RAHMAN et al., 2021; SAYAH et al., 2021; XING et al., 2020; ZHANG et al., 2020).

Através dos estudos que envolviam parâmetros do leucograma, pode-se verificar que leucócitos, neutrófilos, eosinófilos, monócitos e linfócitos podem ser considerados fatores preditivos para o prognóstico da COVID-19.

\subsection{PLAQUETOGRAMA}

As plaquetas apresentaram resultados significativos com níveis mais baixos de pacientes com COVID-19 em relação aos pacientes sem a doença (ESLAMIJOUYBARI et al., 2020; SAYED et al., 2021; SEYIT et al., 2021). Em todos os estudos analisados $(n=6)$ que compararam pacientes graves com casos leves, observou que indivíduos com casos graves de COVID-19 tiveram uma diminuição nos 
níveis de plaquetas em relação a casos leves (BELLAN et al., 2021; FOY et al., 2020; LANINI et al., 2020; LIU et al., 2021; PAKOS et al., 2020; RAHMAN et al., 2021). O mecanismo para trombocitopenia é multifatorial em casos respiratórios agudos graves e refere-se a uma combinação de infecção viral e ventilação mecânica levando a dano endotelial desencadeando ativação, agregação e trombose plaquetária no pulmão, causando grande consumo de plaquetas (ESLAMIJOUYBARI et al., 2020).

O plaquetograma, assim como o eritrograma e o leucograma podem fazer parte de uma ferramenta que indique a evolução, logo nas primeiras horas de internação do paciente com COVID-19, assim auxiliando os profissionais de saúde na tomada de decisão.

\section{CONCLUSÃO}

Com essa revisão da literatura pode-se concluir que os parâmetros: hemoglobina, VCM, RDW, leucócitos, neutrófilos, monócitos, linfócitos e plaquetas apresentaram resultados mais significativos para avaliar pacientes com COVID-19 e assim ter um prognóstico da doença, tornando assim o hemograma completo, um exame simples, rápido e não invasivo, um ótimo recurso de auxílio médico.

\section{AGRADECIMENTOS}

Ao Progama Institucional de Bolsas de Iniciação Científica da Universidade Franciscana (PROBIC/UFN). Ao Programa Estratégico Emergencial de Prevenção e Combate a Surtos, Endemias, Epidemias e Pandemias (CAPES-EPIDEMIAS, Processo: 23038013730202009).

\section{REFERÊNCIAS}

AKMAN, C.; BAKIRDÖĞEN, S. The role of serum inflammatory markers, albumin, and hemoglobin in predicting the diagnosis in patients admitted to the emergency department with a pre-diagnosis of COVID-19. Revista da Associação Médica Brasileira, v. 67, p. 91-96, 2021.

BELLAN, M. et al. Simple Parameters from Complete Blood Count Predict In-Hospital Mortality in COVID-19. Disease Markers, v. 2021, 2021. 


\section{PUFN}

BILGIR, F. et al. Roles of certain biochemical and hematological parameters in predicting mortality and ICU admission in COVID-19 patients. Revista da Associação Médica Brasileira, v. 67, p. 67-73, 2021.

ESLAMIJOUYBARI, M. et al. Neutrophil-to-Lymphocyte and Platelet-to-Lymphocyte Ratios in COVID-19 Patients and Control Group and Relationship with Disease Prognosis. Caspian Journal of Internal Medicine, v. 11, n. Suppl 1, p. 531, 2020. FOY, Brody H. et al. Association of red blood cell distribution width with mortality risk in hospitalized adults with SARS-CoV-2 infection. JAMA Network Open, v. 3, n. 9, p. e2022058-e2022058, 2020.

GALLAND, J. et al. White blood count, D-dimers, and ferritin levels as predictive factors of pulmonary embolism suspected upon admission in noncritically ill COVID-19 patients: The French multicenter CLOTVID retrospective study. European Journal of Haematology, 2021.

GEROTZIAFAS, G. T. et al. Derivation and validation of a predictive score for disease worsening in patients with COVID-19. Thrombosis and haemostasis, v. 120, n. 12, p. 1680-1690, 2020.

GHWEIL, A. A. et al. Characteristics, outcomes and indicators of severity for COVID19 among sample of ESNA Quarantine Hospital's Patients, Egypt: a retrospective study. Infection and Drug Resistance, v. 13, p. 2375, 2020.

KILERCIK, M. et al. A new haematocytometric index: Predicting severity and mortality risk value in COVID-19 patients. Plos one, v. 16, n. 8, p. e0254073, 2021.

LANINI, S. et al. COVID-19 disease-temporal analyses of complete blood count parameters over course of illness, and relationship to patient demographics and management outcomes in survivors and non-survivors: a longitudinal descriptive cohort study. PloS one, v. 15, n. 12, p. e0244129, 2020.

LINSSEN, J. et al. A novel haemocytometric COVID-19 prognostic score developed and validated in an observational multicentre European hospital-based study. Elife, v. 9, p. e63195, 2020.

LIU, H. et al. Development and validation of a risk score using complete blood count to predict in-hospital mortality in COVID-19 patients. Med, v. 2, n. 4, p. 435-447. e4, 2021. 
NALBANT, A. et al. Can the neutrophil/lymphocyte ratio (NLR) have a role in the diagnosis of coronavirus 2019 disease (COVID-19)?. Revista da Associação Médica Brasileira, v. 66, p. 746-751, 2020.

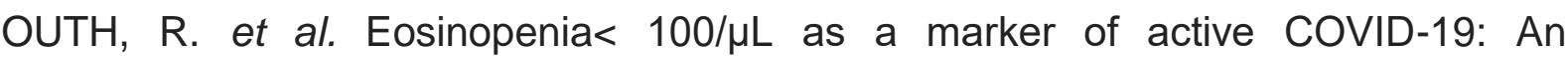
observational prospective study. Journal of Microbiology, Immunology and Infection, v. 54, n. 1, p. 61-68, 2021.

OZBALAK, M. et al. Initial complete blood count score and predicting disease progression in COVID-19 patients. American journal of blood research, v. 11, n. 1, p. $77,2021$.

PAKOS, I. S. et al. Characteristics of peripheral blood differential counts in hospitalized patients with COVID-19. European Journal of Haematology, v. 105, n. 6, p. 773-778, 2020.

RAHMAN, Tawsifur et al. Development and Validation of an Early Scoring System for Prediction of Disease Severity in COVID-19 using Complete Blood Count Parameters. IEEE Access, 2021.

SAYAH, W. et al. Interleukin-6, procalcitonin and neutrophil-to-lymphocyte ratio: Potential immune-inflammatory parameters to identify severe and fatal forms of COVID-19. Cytokine, v. 141, p. 155428, 2021.

SAYED, A. A. A. et al. The use of neutrophil-to-lymphocyte ratio (NLR) as a marker for COVID-19 infection in Saudi Arabia: A case-control retrospective multicenter study. 2021.

SEYIT, M. et al. Neutrophil to lymphocyte ratio, lymphocyte to monocyte ratio and platelet to lymphocyte ratio to predict the severity of COVID-19. The American journal of emergency medicine, v. 40, p. 110-114, 2021.

XING, Y. et al. Analysis of factors for disease progression in 61 patients with COVID19 in Xiaogan, Hubei, China. Eur Rev Med Pharmacol Sci, v. 24, n. 23, p. $12490-$ 12499, 2020.

ZHANG, H. et al. Clinical and hematological characteristics of 88 patients with COVID19. International journal of laboratory hematology, v. 42, n. 6, p. 780-787, 2020. 